\title{
Limits to Error Correction in Quantum Chaos
}

\author{
P G Silvestrov, ${ }^{12} \mathrm{H}$ Schomerus, ${ }^{1} *$ and C W J Beenakker ${ }^{1}$ \\ 'Instituut Lorentz Untversitelt Leiden PO Box 95062300 RA Leiden The Netherlands \\ ${ }^{2}$ Budker Institute of Nuclear Physics 630090 Novosibirsk Russia
}

(Recerved 21 December 2000)

\begin{abstract}
We study the correction of errors that have accumulated in an entangled state of spins as a re sult of unknown local variations in the Zeeman energy $(B)$ and spin-spin interaction energy $(J)$ A nondegenerate code with error rate $\kappa$ can recover the original state with high fidelity within a time $t_{\mathrm{R}} \approx \hbar \kappa^{1 / 2} / \max (B, J)$ - independent of the number of encoded qubits Whether the Hamiltonian is chaotic or not does not affect this time scale, but it does affect the complexity of the error-correcting code
\end{abstract}

DOI $101103 /$ PhysRevLett 865192

In classical mechanics, chaos severely limits the operation of a reversible computer [1] Any uncertanty in the intial conditions is magnified exponentrally by chaotic dynamics, rendering the outcome of the computation unpredictable This is why practical computational schemes are irreversible Dissipation suppresses chaos and makes the computation robust to enors [2] A quantum computer does not have this option, it relies on the reversible unitary evolution of entangled quantum mechanical states, which does not tolerate dissipation [3] This invites the question $[4,5]$ of what limitations quantum chaos might pose on quantum computing

To answer this question one needs to consider the possibilities and restrictions of quantum-error coirection [6] Errors can occur due to interaction with the envilonment (errors of decoherence) and due to uncertanty in the un1tary evolution (unitary errors) The original state can be recovered reliably if the errors involve at most a fraction $\kappa \leqslant 01$ of the total number of qubits The corresponding maximal tıme durıng which errors may be allowed to accumulate (the recovery time $t_{\mathrm{R}}$ ) is easy to find if different qubits are affected independently That may be a reasonable assumption for certain mechanisms of decohetence and also for unitary errors resultıng from an uncertain single-particle Hamiltonian Uncertainttes in the interactions among the qubits pose a more complex problem [7]

Georgeot and Shepelyansky [4] studied this problem for a model Hamiltonian of $N$ interacting spins that exhibits a transition from regular dynamics (nearly isolated spins) to chaotic dynamics (strongly coupled spins) They concluded for the chaotic regime that $t_{\mathrm{R}}$ goes to zero $\propto 1 / N$ for large $N$, but their analysis did not incorporate the opt1mal error-correcting procedure We assume a good (nondegenerate) error-correcting code and obtain a recovery tıme of the order of the inverse energy unceltanty per spınirrespective of the number of encoded qubits By consideing both phase-shift and spin-flip eriors we find that $t_{\mathrm{R}}$ is insensitive to whether the Hamiltonian is chaotic or not (The authors of Refs [4,5] arrived at the opposite conclusion that $t_{\mathrm{R}}$ incieases strongly when chaos is suppressed, but they took only spin-flip errors into account )
PACS numbers $0367 \mathrm{Lx} 0545 \mathrm{Mt} 2460 \mathrm{Lz}, 7440+\mathrm{k}$

The absence of chaos can be used to reduce the complexity of the code, in that a classical error-correcting code suffices for the majority of the errors in the regime of regular dynamics

The Hamiltonian $H$ under consideration describes $N$ coupled spins $\boldsymbol{\sigma}_{n}=\left(\sigma_{n}^{x}, \sigma_{n}^{\gamma}, \sigma_{n}^{z}\right)$ on a lattice in a magnetic field $\mathbf{B}_{n}=\left(B_{n}^{x}, B_{n}^{y}, B_{n}^{z}\right)$,

$$
H=\sum_{n} \mathbf{B}_{n} \quad \boldsymbol{\sigma}_{n}+\sum_{n \neq m} \boldsymbol{\sigma}_{n} \quad \mathbf{J}_{n m} \quad \boldsymbol{\sigma}_{m}
$$

A spin $n$ interacts with $d$ neighboring spins $m$ via the matrix $\mathbf{J}_{n m}$ The spin could be a nuclear spin or the spin of an electron confined to a quantum dot, in the context of solid-state based proposals for quantum computing [8-10] More generally, the spin could be a representation of a two-level system (for example, in the context of the ion-trap quantum computer [11]) We assume that $\mathbf{B}_{n}$ and $\mathbf{J}_{n m}$ fluctuate independently from site to site, with zero mean and variance $\overline{\left|\mathbf{B}_{n}\right|^{2}}=B^{2}$ and $\sum_{\alpha \beta} \overline{\left(J_{n m}^{\alpha \beta}\right)^{2}}=J^{2}$ (provided $m$ is one of the $d$ neighbors of $n$, otherwise $\left.\mathbf{J}_{n m}=0\right) \quad$ We denote by $U=\left(B^{2}+2 d J^{2}\right)^{1 / 2}$ the root-mean-square energy uncertainty per spin

A state $\psi_{0}$ evolves in time according to $\psi(t)=e^{-\imath H t} \psi_{0}$ (setting $\hbar \equiv 1$ ) We assume that we do not know the parameters of the Hamiltonian, and use quantum-error correction to recover $\psi_{0}$ from $\psi(t)$ [12] Let $\psi_{0}$ lie in the code space of a nondegenerate error-correctung code [6] The code space is a $2^{M}$ dimensional subspace of the full $2^{N}$ dimensional Hilbert space, such that

$$
\left\langle\psi_{0}\left|\sigma_{n_{1}}^{\alpha_{1}} \sigma_{n_{1}}^{\alpha,} \quad \sigma_{n_{k}}^{\alpha_{h}}\right| \psi_{0}^{\prime}\right\rangle=0, \quad 1 \leq k \leq 2 K,
$$

for any two (possibly identical) states $\psi_{0}, \psi_{0}^{\prime}$ in the code space and any product of up to $2 K$ Paulı matrices $\sigma_{n}^{\alpha}$ (acting on different spins $n_{1}, n_{2}$, ) The number $M$ is the number of qubits encoded in $N$ spins The number $K$ is the number of errors that the code can correct, wheie the application of $\sigma_{n}^{\lambda}, \sigma_{n}^{3}$, or $\sigma_{n}^{z}$ to any of the $N$ spins counts as one eno1 The iatio $M / N=\rho$ is the bit rate of the code and the ratio $K / N=\kappa$ the enor rate

Enol conlection is successful if $\psi(t)$ lies in the error space of $\psi_{0}$, which is the subspace spanned by the state $\psi_{0}$ 
and the states derived from $\psi_{0}$ by making up to $K$ errors. The operator $\mathcal{P}$ projects onto the error space. Explicitly, $\mathcal{P}=\sum_{p=0}^{K} \mathcal{P}_{p}$, with

$$
\mathcal{P}_{p}=\sum_{\{n, \alpha\}} \frac{1}{p !} \sigma_{n_{1}}^{\alpha_{1}} \ldots \sigma_{n_{p}}^{\alpha_{p}}\left|\psi_{0}\right\rangle\left\langle\psi_{0}\right| \sigma_{n_{1}}^{\alpha_{1}} \ldots \sigma_{n_{p}}^{\alpha_{p}}
$$

The symbol $\sum_{\{n, \alpha\}}$ indicates a summation over the $n_{\imath}$ 's and $\alpha_{i}$ 's, with the restriction that the indices $n_{1}, n_{2}, \ldots$ should be all distinct. (The indices $\alpha_{1}, \alpha_{2}, \ldots$ need not be distinct.) The norm

$$
F(t)=|\mathcal{P} \psi(t)|^{2}=\left\langle\psi_{0}\left|e^{l H t} \mathcal{P} e^{-t H t}\right| \psi_{0}\right\rangle
$$

of the projected state is the probability of successful error correction after a time $t$. It is the "fidelity" of the recovered state [6]. The recovery time $t_{\mathrm{R}}$ can be defined as the time at which the fidelity has dropped from 1 to $1 / 2$.

We assume that the error-correcting code is "good," meaning that $\rho$ and $\kappa$ tend to a nonzero value as $N \rightarrow \infty$. Good quantum-error correcting codes exist, but their construction for large $N$ is a complex problem [13-18]. Our strategy will be to derive a lower bound to $F$ and $t_{\mathrm{R}}$ that does not use any properties of the code beyond the nondegeneracy condition (2), so that we can avoid an explicit construction. An alternative approach would be to abandon the requirement of a good code, and keep the number $M$ of encoded qubits fixed as the total number of spins $N$ goes to infinity. One can then use the technique of concatenation [6] to construct codes that are safe for a large number of errors at the expense of a vanishingly small bit rate $\rho$. (See Ref. [19] for such a calculation in the case $M=1$.)

Our first step is to decompose the evolution operator $e^{\imath H t}=\sum_{k=0}^{N} X_{k}$ into operators $X_{k}$ that create $k$ errors. For $k \ll N$ and $t \ll 1 / U$ we may approximate

$$
\begin{aligned}
X_{k}= & X_{0} \sum_{q=0,2,4}^{k} \sum_{\{n, \alpha\}} \frac{(i t)^{k-q / 2}}{(k-q) !(q / 2)} \sigma_{n_{1}}^{\alpha_{1}} \ldots \sigma_{n_{k}}^{\alpha_{k}} \\
& \times J_{n_{1} n_{2}}^{\alpha_{1} \alpha_{2}} \ldots J_{n_{q-1} n_{q}}^{\alpha_{q-1} \alpha_{q}} B_{n_{q+1}}^{\alpha_{q+1}} \ldots B_{n_{k}}^{\alpha_{k}} \\
X_{0}= & \exp \left[-\frac{1}{2} t^{2} \sum_{n}\left|\mathbf{B}_{n}\right|^{2}-t^{2} \sum_{n \neq m} \sum_{\alpha, \beta}\left(J_{n m}^{\alpha \beta}\right)^{2}\right] .
\end{aligned}
$$

The approximation consists of neglecting terms in the exponent of order $k(U t)^{2}$ and $N(U t)^{4}$, relative to the terms retained of order $N(U t)^{2}$. We may write $X_{0} \approx$ $\exp \left[-\frac{1}{2} N(U t)^{2}\right]$, neglecting fluctuations in the exponent that are smaller by a factor of $1 / \sqrt{N}$.

We next substitute the decomposition of $e^{t H t}$ in error operators into the fidelity (4),

$$
F(t)=\sum_{p=0}^{K} \sum_{k, k^{\prime}=0}^{N}\left\langle X_{k} \mathcal{P}_{p} X_{k^{\prime}}^{*}\right\rangle
$$

where we have abbreviated $\langle\cdots\rangle=\left\langle\psi_{0}|\cdots| \psi_{0}\right\rangle$. To simplify this expression, we take the average over the random variations in the $B_{n}$ 's and $J_{n m}$ 's. (We will show later that statistical fluctuations around the average are insignificant.) Only the terms with $k=k^{\prime}$ contribute to the average. The terms with $p+k \leq 2 K$ can be simplified further, since they contain at most $2 K$ Pauli matrices. In view of Eq. (2), these expectation values vanish unless the product of Pauli matrices reduces to a $c$ number, which requires $p=k$. Hence the average fidelity can be written as $\bar{F}=F_{1}+F_{2}$, with

$$
\begin{aligned}
& F_{1}=\sum_{p=0}^{K} \sum_{\{n, \alpha\}} \frac{1}{p !} \overline{\left.\left\langle X_{p} \sigma_{n_{1}}^{\alpha_{1}} \cdots \sigma_{n_{p}}^{\alpha_{p}}\right\rangle\right|^{2}}, \\
& F_{2}=\sum_{p=0}^{K} \sum_{k=2 K+1-p}^{N} \sum_{\{n, \alpha\}} \frac{1}{p !} \overline{\left|\left\langle X_{k} \sigma_{n_{1}}^{\alpha_{1}} \cdots \sigma_{n_{p}}^{\alpha_{p}}\right\rangle\right|^{2}} .
\end{aligned}
$$

The expectation values in $F_{1}$ are evaluated by substituting Eq. (5) and extracting the terms that reduce to a $c$ number,

$$
F_{1}=e^{-N(U t)^{2}} \sum_{p=0}^{K} \sum_{q=0,2,4}^{p} \frac{\left(N B^{2} t^{2}\right)^{p-q}}{(p-q) !} \frac{\left(2 N d J^{2} t^{2}\right)^{q / 2}}{(q / 2) !}
$$

For $K \gg 1$ we may approximate $e^{-x} x^{k} / k ! \approx$ $(2 \pi x)^{-1 / 2} \exp \left[-(k-x)^{2} / 2 x\right]$ and replace the sums in Eq. (10) by integrals. The result is

$$
\begin{gathered}
F_{1}=\frac{1}{2}+\frac{1}{2} \operatorname{erf}\left[\left(t_{\mathrm{R}}-t\right) / \Delta t\right] \\
t_{\mathrm{R}}=\sqrt{\frac{\kappa}{B^{2}+4 d J^{2}}}, \quad \Delta t=\sqrt{\frac{1}{2 N} \frac{\sqrt{B^{2}+8 d J^{2}}}{B^{2}+4 d J^{2}}},
\end{gathered}
$$

with $\operatorname{erf}(x)=2 \pi^{-1 / 2} \int_{0}^{x} e^{-y^{2}} d y$ the error function. Corrections to $t_{\mathrm{R}}$ and $\Delta t$ arising from the approximations made in Eqs. (5) and (6) are smaller by a factor $\kappa$. The expectation values in $F_{2}$ depend specifically on $\psi_{0}$, hence on the way in which $M$ qubits are encoded in $N$ spins. Since $F_{2} \geq 0$ we have a lower bound $\bar{F} \geq F_{1}$ on the fidelity that is code independent within the class of nondegenerate error-correcting codes.

For $N \rightarrow \infty$ the time dependence of Eq. (11) approaches the step function $\theta\left(t_{\mathrm{R}}-t\right)$. The threshold $t_{\mathrm{R}}$ is independent of $N$, while the width $\Delta t$ of the transition vanishes as $N^{-1 / 2}$ (solid curves in Fig. 1). These are results for the ensemble-averaged fidelity, but since the variance is bounded by $0 \leq \operatorname{var} F \leq \bar{F}(1-\bar{F})$ the fluctuations are insignificant except in the narrow transition region. The stepfunction behavior of the fidelity also implies that the positive code-dependent term $F_{2}$ that we have not included in Fig. 1 satisfies $\lim _{N \rightarrow \infty} F_{2} \rightarrow 0$ for $t<t_{\mathrm{R}}$ (since $F_{1}+F_{2} \leq 1$ and $F_{1} \rightarrow 1$ for $t<t_{\mathrm{R}}$ ). Any code dependence of the fidelity can therefore appear only for times greater than $t_{\mathrm{R}}$.

The independence of the recovery time on the number $M$ of encoded qubits disagrees with Refs. [4,5]. These authors calculated the squared overlap $\left|\left\langle\psi_{0} \mid \psi(t)\right\rangle\right|^{2} \approx X_{0}^{2}$ of 


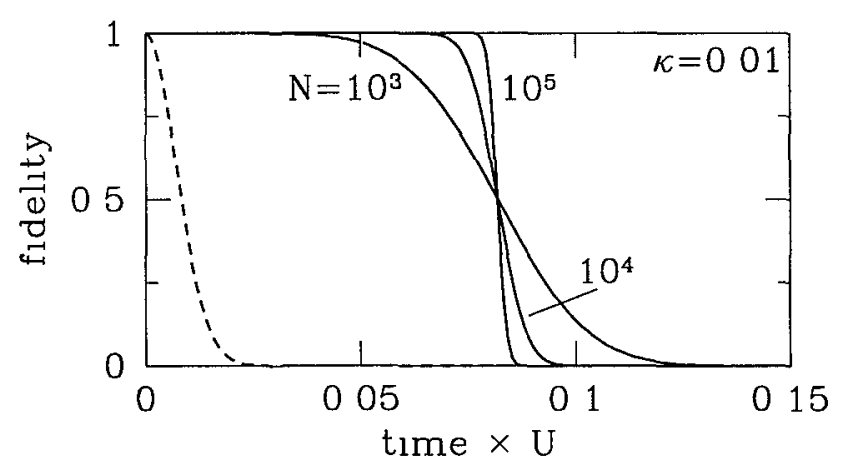

FIG 1 Solid curves time dependence of the lower bound $F_{1}$ to the ensemble-averaged fidelity, calculated from $\mathrm{Eq}(11)$ for error rate $k=001$ and three values of $N$ (We took $B^{2}=2 d J^{2}$, so that the root-mean-squared energy uncertainty per spin $U$ is equally divided between Zeeman energy and interaction energy) The dashed curve shows (for $N=10^{4}$ ) the squared overlap $X_{0}^{2}=\exp \left[-N(U t)^{2}\right]$ between initial and final states In all these curves the number $M$ of encoded qubits is a fixed fraction $\rho$ of the total number of spins $N$

the time-dependent state with the original state, and argued that the original state would be effectively lost once this overlap is $\ll 1$ However, the original state can be recovered even when this overlap has become exponentially small, if a good error-conecting code is used (compare dashed and solid curves in $F_{1} g$ 1) The recovery time is increased by a factor $\sqrt{\kappa M}$, with an overhead of $1 / \rho$ spins per encoded qubit

We find that $t_{\mathrm{R}}$ at a given $U$ is insensitive to the relative magnitude of $B$ and $J$, and hence insensittve to whether the Hamiltonian is chaotic or not This conclusion may seem surprising in view of the fact that the eigenstates aie completely different in the chaotic and regular regimes [4] For $J<B / N$ the eigenstates of the total Hamiltonian $H$ are a superposition of a small number of eigenstates of the noninteracting pait $\sum_{n} \mathbf{B}_{n} \cdot \boldsymbol{\sigma}_{n}$ This number (known as the paiticipation 1atio) increases with increasing $J$, and when $J \approx B$ it becomes of the same order as the dimension $2^{N}$ of the entire Hilbert space (See Ref [20] for a description of the onset of quantum chaos in systems with random two-body interactions ) As we will now discuss, the reason that a small participation ratio does not improve the fidelity is that it counts spin-flip errors but not phase-shift errors For the same reason, suppression of chaos does help to reduce the complexity of the etror-correcting code

The three Paul matrices correspond to three types of errors spin flips $\left(\sigma^{\lambda}\right)$, phase shifts $\left(\sigma^{z}\right)$, and a combination of the two $\left(\sigma^{y}=\imath \sigma^{x} \sigma^{z}\right)$ The complexity of the code is reduced substantially if there is only one type of error to coirect (One can then use a code for classical bits, such as the Hamming code [6] ) Suppose that we seek to suppress spin-flup errors, of either type $\sigma^{\lambda}$ or $\sigma^{y}$ To this end we impose on the spins a known unıform magnetic field in the $z$ direction, with Zeeman eneigy $B_{0}$ that is large compared to the magnitude $U$ of the random energy vaisations The new Hamilonian is $H+H_{0}$, with $H$ given by
Eq (1) and $H_{0}=B_{0} \sum_{n} \sigma_{n}^{z}$ Since $B_{0}$ is known we can undo the evolution of a state due to $H_{0}$ by applying the operator $e^{\imath H_{0} t}=\prod_{n}\left(\cos B_{0} t+\imath \sigma_{n}^{z} \sin B_{0} t\right)$ Any remaining deviation of $\psi(t)$ from $\psi_{0}$ has to be dealt with by the errol-correcting code, with projection operator $\mathcal{P}$ The fidelity of the corrected state is $F(t)=\left|\mathcal{P} G(t) \psi_{0}\right|^{2}$, where the evolution operator $G$ is defined by

$$
G(t)=e^{\imath H_{0} t} e^{-t\left(H+H_{0}\right) t}=\mathcal{T} \exp \left(-\imath \int_{0}^{t} H\left(t^{\prime}\right) d t^{\prime}\right)
$$

[The notation $\mathcal{T}$ indicates time ordering of the operators $\left.H(t)=e^{t H_{0} t} H e^{-t H_{0} t}\right]$

For $B_{0} t \gg 1$ we may replace $H(t)$ by its time average over the interval $\left(t, t+1 / B_{0}\right)$ The terms containing a single $\sigma^{x}$ or $\sigma^{y}$ average out to zero and we are left with

$$
\begin{aligned}
G(t) & =e^{-\imath t\left(H_{\|}+H_{\perp}\right)}, \quad H_{\|}=\sum_{n} B_{n}^{z} \sigma_{n}^{z}+\sum_{n \neq m} J_{n m}^{z z} \sigma_{n}^{z} \sigma_{m}^{z}, \\
H_{\perp} & =\sum_{n \neq m} \frac{1}{2}\left(J_{n m}^{x x}+J_{n m}^{y y}\right)\left(\sigma_{n}^{x} \sigma_{m}^{x}+\sigma_{n}^{y} \sigma_{m}^{y}\right)
\end{aligned}
$$

(We have assumed $J_{n m}^{x y}=J_{n m}^{y x}$, so that the mixed terms $\sigma_{n}^{x} \sigma_{m}^{y}$ cancel ) The time dependence of the fidelity is again given by Eq (11), with $B^{2}=\overline{\left(B_{n}^{z}\right)^{2}}$ and $J^{2}=\overline{\left(J_{n m}^{z z}\right)^{2}}+\frac{1}{4} \overline{\left(J_{n m}^{x \lambda}+J_{n m}^{y y}\right)^{2}}$ The recovery time $t_{\mathrm{R}}$ depends only weakly on the ratio $J / B$ The 1elative numbei of phase-shift and spin-flip enors, however, depends stiongly on this ratio Indeed, if one would use a code that corrects up to $K_{\|}$eirols from $\sigma^{z}$ and up to $K_{\perp}$ errors from $\sigma^{x}$ or $\sigma^{3}$, then the maximal $t_{\mathrm{R}}$ (at fixed $K_{\|}+K_{\perp}$ ) is reached for $K_{\perp} / K_{\|}=4 d J^{2} / B^{2} \quad$ For $J \ll B$ one has $K_{\perp} \ll K_{\|}$, so that a classical error-correcting code suffices for the majority of errors

Before concluding we briefly consider the case that the parameteis $\mathbf{B}_{n}$ and $\mathbf{J}_{n m}$ in the Hamilonian are not only unknown but also time dependent The result (10) still holds if we replace $(B t)^{2}$ by the correlator $b(t)=\int_{0}^{t} d t^{\prime} \int_{0}^{t} d t^{\prime \prime} \overline{\mathbf{B}_{n}\left(t^{\prime}\right)} \quad \mathbf{B}_{n}\left(t^{\prime \prime}\right)$, and similarly replace $(J t)^{2}$ by $J(t)=\int_{0}^{t} d t^{\prime} \int_{0}^{t} d t^{\prime \prime} \sum_{\alpha \beta} \overline{J_{n m}^{\alpha \beta}\left(t^{\prime}\right) J_{n m}^{\alpha \beta}\left(t^{\prime \prime}\right)}$ For a short-time correlation one has $b(t)=b_{0}|t|$, $J(t)=J_{0}|t|$ This leads for $K \gg 1$ to the fidelity

$$
\begin{gathered}
F_{1}=\frac{1}{2}+\frac{1}{2} \operatorname{erf}\left[\left(t_{\mathrm{R}}-t\right) / \Delta t\right], \\
t_{\mathrm{R}}=\frac{\kappa}{b_{0}+4 d_{j_{0}}}, \quad \Delta t=\sqrt{\frac{2 \kappa}{N}} \frac{\sqrt{b_{0}+8 d_{J_{0}}}}{\left(b_{0}+4 d_{j_{0}}\right)^{3 / 2}}
\end{gathered}
$$

The recovery time now depends linearly on the etror rate $\kappa$, but it remains $N$ independent The next step towards fault-tolerant computıng, which we leave for a future investigation, would be to include in the Hamiltonian a part with a known time dependence (That part would switch on and off the coupling between pairs of spins in a prescribed way, in oider to realize the logical gates ) 
In conclusion, we have derived a code-independent lower bound for the fidelity $F$ of a state that has been recovered after a unitary evolution for a time $t$ in an unknown random magnetic field and spin-spin interaction For a large system the transition from $F=1$ to $F=0$ occurs abruptly at a time $t_{\mathrm{R}}$ that is independent of the total number of spins $N$ and the number of encoded qubits $M \quad$ The magnitude of $t_{\mathrm{R}}$ is set by the inverse eneigy uncertainty per spin, regardless of whether the spins are nearly isolated or strongly coupled The suppression of chaos that occurs when the spins are decoupled does not improve the fidelity, because of the persistence of phase-shift errors Spin-flip errors can be suppressed, and this helps to reduce the complexity of the error-correcting code

In this work we have concentrated on the recovery from unitary errors One might question whether suppression of quantum chaos improves the fidelity for recovery from errors of decoherence, in particulat in view of the "hypersensitivity to perturbation" observed in computer simulations of systems with a chaotic dynamics [21,22] This question presents itself as an interesting topic for future research

We thank $P$ Zoller for a valuable discussion This work was supported by the Dutch Science Foundation NWO/FOM PG S acknowledges the support of the RFBR Grant No 98-02-17905

*Present address Max-Planck-Institut fur Physık komplexer Systeme, Nothnitzer Str 38, 01187 Dresden, Germany

[1] C H Bennett and R Landauer, Scr Am 253, 38 (1985)

[2] R Landauer, Phys Lett A 217, 188 (1996)

[3] J Gruska, Quantum Computing (McGraw-Hıll, London, 1999), M A Nielsen and I L Chuang, Quantum Computation and Quantum Information (Cambridge University Press, Cambridge, 2000)

[4] B Georgeot and D L Shepelyansky, Phys Rev E 62, 3504 (2000), 62, 6366 (2000)
[5] V V Flambaum, Aust J Phys 53, 489 (2000)

[6] For an introduction and review of quantum-error correction, see J Preskill's lecture notes at http //theory caltech edu/people/preskill

[7] J Gea-Banacloche, Phys Rev A 57, R1 (1998)

[8] V Privman, I D Vagner, and G Kventsel, Phys Lett A 239, 141 (1998)

[9] B E Kane, Nature (London) 393, 133 (1998)

[10] D Loss and D P DiVincenzo, Phys Rev A 57, 120 (1998)

[11] J I Cirac and P Zoller, Phys Rev Lett 74, 4091 (1995)

[12] In special cases one can use a spin-echo technique, instead of quantum-error correction, to invert the unitary evolution This works for the Hamiltonian of Refs [4,5], which has the special form $H=\sum_{n} B_{n} \sigma_{n}^{z}+\sum_{\mu \nu} J_{\mu \nu} \sigma_{\mu}^{x} \sigma_{\nu}^{x}$, the spins $\mu$ and $\nu$ belonging to two disjunct sublattices The spin echo is prepared by the unitary operator $S=\prod_{\mu \nu} \sigma_{\mu}^{x} \sigma_{\nu}^{y}$, which satisfies $S H S=-H$ The state $S \psi_{0}$ is allowed to evolve under the action of $H$ for a time interval $t$, after which $S$ is applied a second time After another time $t$ the original $\psi_{0}$ is recovered, since $e^{-t H t} S e^{-\imath H t} S=1$ A variation on this spin-echo technique is the " $2 \pi k$ " method discussed by G P Berman, G D Doolen, G V López, and V I Tsifrinovich, Phys Rev A 61, 042307 (2000)

[13] A R Calderbank and P W Shor, Phys Rev A 54, 1098 (1996)

[14] A M Steane, Proc R Soc London A 452, 2551 (1996)

[15] D Gottesman, Phys Rev A 54, 1862 (1996)

[16] A Ekert and C Macchravello, Phys Rev Lett 77, 2585 (1996)

[17] A R Calderbank, E M Rains, P W Shor, and N J A Sloane, Phys Rev Lett 78, 405 (1997)

[18] E Knill, R Laflamme, and L Viola, Phys Rev Lett 84, $2525(2000)$

[19] J Gea-Banacloche, Phys Rev A 62, 062313 (2000)

[20] P G Silvestrov, Phys Rev E 58, 5629 (1998), X Leyronas, P G Silvestrov, and C W J Beenakker, Phys Rev Lett 84, 3414 (2000)

[21] R Schack and C M Caves, Phys Rev E 53, 3257 (1996)

[22] P H Song and D L Shepelyansky, Phys Rev Lett 86, 2162 (2001), P H Song, quant-ph/0010075 